\title{
LA ESCUELA PÚBLICA ANTE LA PRESIÓN POR LA COMPETITIVIDAD: ¡USEMOS LA COLABORACIÓN COMO ANTÍDOTO!
}

\author{
Serafín Antúnez \\ Universidad de Barcelona
}

\begin{abstract}
Due to the increasing pressure within the teaching field there is a new view that proposes collaboration amongst schools in order to release this pressure in a creative common way. The proposed collaboration explains the main characteristics and requirements including the scientific justification and basing, also identifying some antecedents and present achievements. Moreover, it offers lines to guide the actions showing the way for the development and improvement as well as taking into account the necessary precautions and conditions so as to develop the culture of the collaboration to give power and dignity to state schools.

RESUMEN. Ante la configuración de un nuevo panorama en el desarrollo del trabajo docente marcado por la presión por la competitividad, se propone un modelo de colaboración entre centros que permita responder a esas tensiones de forma creativa y solidaria.

El modelo propuesto explícita las características y requisitos principales, así como su justificación y fundamentación científica. Igualmente, identifica algunos antecedentes asi como realizaciones actuales. Así mismo, ofrece pautas para guiar las acciones encaminadas al desarrollo y la mejora de la colaboración, considerando las precauciones y condiciones que es necesario tener presente para desarrollar la cultura de la colaboración con el propósito de potenciar y dignificar la escuela pública.
\end{abstract}

\section{A modo de introducción}

Quienes trabajamos como enseñantes somos, en general, bien conscientes de que nuestra tarea tiene como objetivo fundamental proporcionar la mejor ayuda que seamos capaces a nuestros estudiantes. Además, la gran mayoría concebimos nuestro trabajo como una práctica claramente conducente a satisfacer el derecho que tienen a recibir una educación escolar que esté guiada por los principios normativos de respeto, equidad, igualdad de oportunidades y justicia.

El afán, pues, por hacer bien las cosas, por ser diligentes en nuestro trabajo, conscientes de su importancia, no es un acontecimiento nuevo o una moda reciente entre nosotros sino una práctica común y bien extendida que viene de antiguo y que tratamos de desarrollar responsablemente. 
Sin embargo, en estos últimos años, el escenario en el que nos movemos está configurándose de forma diferente a causa de una serie de elementos nuevos que conforman un panorama distinto. Me refiero a un conjunto de factores y presiones que nos conducen a desarrollar nuestro trabajo en un marco desigual o menos conocido. Se nos plantea responder a unos requerimientos, en ocasiones, también nuevos o distintos que nos producen múltiples tensiones y que nos conducen a desarrollar nuestro cometido acompañados de parcelas de incertidumbre cada vez mayores.

De entre todos estos factores vamos a destacar algunos. Se nos presentan en forma de discursos, en forma de hechos, de prácticas, de indicios, en forma de amenazas, pero todos ellos se complementan y parecen ajustar muy bien como piezas de un mismo rompecabezas.

\section{Los discursos; o de la pasión por la calidad a sufrir una pasión a causa de la calidad.}

El primero de los discursos tiene que ver con la presión por que desarrollemos nuestro trabajo con arreglo a criterios de calidad.

Se nos dice insistentemente: "(deben intentar que la educación que Vdes. procuran sea cada vez de mayor calidad: los estudiantes, el país, los nuevos tiempos, el "compromiso"? con Europa.. lo requieren! Se apela para ello a nuestra profesionalidad o a nuestro compromiso social... Ahora bien, ¿en qué consiste esa educación de calidad que se nos pide?, ¿respecto a qué criterios se establece?, ¿con qué intenciones explícitas y ocultas?, ¿en qué marco de interpretación? El uso pertinaz del término se ha introducido profundamente en las prédicas de los responsables y de quienes diseñan las políticas educativas, en el léxico habitual de quienes las ejecutan y evalúan y también en el de quienes investigan y proponen en materia de educación escolar. La literatura generada a partir del movimiento de las Escuelas Eficaces, las directrices y políticas que pretenden promoverse desde la Administración Educativa en España en relación a la calidad (título IV de la LOGSE), el "Proyecto de las 77 medidas" u otras propuestas concretas (López Rupérez, 1997) son algunos ejemplos.

El segundo discurso se relaciona con la idea de eficacia. En aras a la asunción y el compromiso con las nociones de calidad, eficiencia o excelencia se nos pide a los enseñantes y también a los establecimientos escolares, en tanto que organizaciones, que seamos eficaces ya que (i) hay que justificar razonablemente el uso de las parcelas de autonomía que, según parece, van a ir en aumento, (ii) hay que dar cuenta del uso de los recursos que se ponen a nuestra disposición. Todo esto no es nuevo, ha existido siempre, pero en la actualidad se presenta con una intensidad mayor, con una contundencia inhabitual.

El tercero se refiere a la presión por que seamos competitivos. La noción de competitividad, trasladada directamente del léxico de las organizaciones comerciales o industriales y aún de las políticas económicas de los países, se plantea no tanto como el hecho de adquirir capacidad para abordar un determinado propósito o como sana emulación, seguramente recomendable, sino como una manifestación con fuertes connotaciones de rivalidad y disputa. 
Como consecuencia, la sana pasión por la calidad y por la eficiencia, que todos compartimos, es decir, la inclinación preferente, viva y vehemente por la mejora, a través de la revisión de los procesos y los resultados, está dando lugar a otra pasión: la que comporta el acto de padecer y sufrir un calvario escolar ante unas exigencias que no siempre van acompañadas de recursos y de algunas evidencias amenazantes.

\section{Las evidencias}

Otros hechos, prácticas e indicios ayudan a completar estos discursos y a configurar aquel panorama complejo. Por ejemplo, la posibilidad, ya no tan lejana, en nuestro país de que los procesos de evaluación externa de los establecimientos escolares, siempre necesarios, deriven en un afán por establecer clasificaciones, ordenar o comparar a los centros entre sí. Son prácticas ya habituales en otros países. Recuérdense algunas épocas recientes en el Reino Unido o, actualmente, las propuestas en algunos países de Iberoamérica, $o$, también las prácticas en otros estados en los que el prestigio social de la educación escolar pública está fuera de toda duda (Le Monde de l'Éducation, 1995). Y, por otra parte, la posible amenaza de que la asignación de los recursos a cada centro pueda estar algún día en función de los resultados de aquellas evaluaciones sin aclarar previamente si detrás de esta decisión está la de reforzar y ayudar a quienes están en la situación peor o más precaria o con el fin de tener una coartada para justificar su eliminación o cierre.

Por otra parte, es también una evidencia el avance de determinados planteamientos neoliberales en la educación escolar de nuestro país (Angulo, 1995). Es decir, la preponderancia de una corriente de pensamiento y de acción que pone el énfasis en conceptos y valores como libre mercado, privatización, puritanismo o reacción contra ideologías de componentes socialistas. Todo ello origina ciertas dudas entre los enseñantes cuando encuentran respuestas confusas a preguntas como: ¿quién ha dicho que debemos ser competitivos?, ¿por qué ahora precisamente?, ¿con qué fin?

El panorama se compone también de otros hechos e indicios. Por una parte, nuevas presiones y requerimientos derivados de la puesta en marcha de una reforma educativa: en relación al currículo, a la necesidad de actualización científica y didáctica de los docentes, o relativos a la problemática específica de la nueva etapa secundaria obligatoria. Por otra, el descenso de la matrícula en los centros de infantil y primaria y sus consecuencias en forma de cierre progresivo y masivo de aulas y de algunos centros públicos desde hace cinco años, especialmente en algunas zonas.

Así pues, los discursos, las voces, las evidencias y las presiones parecen sugerirnos: (aprendan a moverse en el terreno de la competitividad), (gánense ustedes a sus alumnos), (compitan), (combatan a la competencia), (convenzan a su clientela)

\section{Los efectos y las respuestas}

Este nuevo paisaje, del cual estamos enumerando sólo los aspectos que se relacionan más directamente con el núcleo central de nuestra reflexión, produce desasosiego, inseguridad y desequilibrio entre los enseñantes. Así, nos preguntamos: los 
enseñantes, mis colegas, que trabajan en otros centros ¿qué serán a partir de ahora para mi?: ¿compañeros?, ¿rivales?. ¿El futuro de la escuela pública será especializarse en casos y situaciones extremas?, ¿en la atención a alumnos de necesidades educativas especiales?, ¿en la atención a colectivos minoritarios o marginales?. ¿Entramos en la dinámica de la competivividad o estamos destinados a atender a la marginalidad? (Cruz, 1996). En suma, en la enseñanza pública: ¿debemos colaborar o competir con nuestros colegas y con los demás centros escolares? (Macbeth y otros, 1995).

Ahora bien, ¿qué respuestas estamos dando ante esos interrogantes? Ante tal incertidumbre es evidente que, por ahora, desde el sector público, existe una falta de capacịdad para aglutinar réplicas coordinadas y colegiadas por parte de los establecimientos escolares, los servicios de inspección, los sindicatos, los movimientos asociativos de padres y madres o de estudiantes o de las personas que trabajamos en las universidades.

Los poderes públicos, por su parte, la Administración Educativa, el patrón, en suma, parece manifestar muy poco interés en articular y organizar su propio dispositivo con la intención de encontrar respuestas creativas y eficaces a estas nuevas problemáticas. No suele promocionar ni potenciar su propio aparato de establecimientos y servicios escolares, enfatizando en sus bondades y sus logros y, cuando lo hace, manifiesta conductas pusilánimes y poco convencidas.

Por nuestra parte, en los centros públicos, habrá que reconocerlo, tampoco ha habido una gran preocupación por encontrar caminos nuevos. Hasta hace pocos años los índices de natalidad que garantizaban una demanda escolar abundante y segura, una capacidad operativa escasa de los centros y unos sistemas de evaluación institucional poco adecuados han contribuido a la existencia de cierta desidia y rutinización.

En estos momentos es perceptible y evidente una cierta actitud de renuncia, de claudicación, de impotencia, tal vez de desilusión en ellos. Se diría que ofrecer una mejor educación pública es un propósito al que renunciamos porque lo vemos difícil. Las voces y manifestaciones por mejorar y dignificar la escuela pública son episódicas e inconstantes, ¿qué se ha hecho con esa finalidad desde la manifestación del día 17 de mayo del 97 a favor de la escuela pública? (que correspondía, por cierto, al curso escolar anterior), ¿qué ha pasado desde entonces?

Por otra parte, parece que los intentos de colaboración entre centros fenecen, se debilitan o no parecen necesarios cuando los establecimientos escolares están protegidos por el paraguas protector de la Administración, madre nutricia, no siempre abundante en sus aportaciones, pero constante y segura. Resulta curioso observar como, por ejemplo, los centros asociados al CEPEPC (Col.lectiu d'Escoles per a I'Escola Pública Catalana), de iniciativa privada, la mayoría de ellos creados durante la década de los sesenta, constituidos en asociación en 1978, y, en general, agentes de innovación pedagógica durante muchos años, a partir del momento en que pasan a formar parte de la red de escuelas públicas catalanas, el interés por colaborar entre si se desvanece, la idea de colectivo mengua y las acciones colaborativas ya no son perceptibles.

Estamos atentos por comprobar si este mismo proceso se va a repetir en el caso del colectivo de las ikastolas. Durante muchos años la inmensa mayoría de ellas, en Euskadi, han estado asociadas a su Federación, a través de la cual han realizado man- 
comunadamente acciones muy meritorias en pro de la renovación pedagógica. Recientemente, con motivo de los cambios legislativos en el sistema educativo de aquel país algunos de estos establecimientos escolares han optado por incorporarse a la red de centros públicos. ¿Qué pasará a partir de ahora en ellos?; ¿considerarán que sigue mereciendo la pena trabajar colaborativamente?; ¿seguirá cada ikastola su propio camino, individualmente ya que ahora, instalada en la red pública, cree no necesitar de las demás?

Otros estamentos de la comunidad escolar, en cambio, parecen tener un mayor interés por las acciones mancomunadas. Es el caso de las asociaciones de padres y madres de alumnos, mediante sus federaciones y confederaciones o el de los estudiantes, vinculados también mediante federaciones de asociaciones de alumnos $y$ alumnas.

Algo nos está pasando: ¿claudicación ante un mal inevitable?, ¿desidia?. Sin embargo, a la vez, ante todas estas presiones, nuevos marcos, confusiones e incertidumbres sí que todos y todas compartimos la idea de que la competición entre centros escolares públicos no es la mejor manera, ni siquiera un manera honesta para mejorar la eficacia docente y la calidad de la enseñanza.

\section{5. ¿Cómo hacer frente a esa situación? Una medicina para preservar del mal: la colaboración entre centros}

En estos últimos años son perceptibles algunas reacciones aisladas, asistemáticas e individuales de algunos centros públicos ante esa situación. Si de lo que se trata es dar una respuesta adecuada a los nuevos requerimientos y ese propósito comporta, en primer lugar, darse a conocer, algunos establecimientos escolares han iniciando acciones, a la manera de otras organizaciones industriales, comerciales o de servicios, recurriendo a las estrategias de mercadotecnia más convencionales. Tratan de ganar terreno a los demás, en suma: de competir.

Frente a esta concepción, basada en la rivalidad, en la lucha contra la competencia, planteamos otra basada en cinco factores (Antúnez, 1997): información, transparencia, justicia, compensación de desigualdades y colaboración entre centros.

En otras ocasiones nos hemos referido a cómo desarrollar los dos primeros (Antúnez, 1997), a partir de destacar algunas evidencias y sus consecuencias. Nuestra reflexión de hoy quiere centrarse en el último de estos cinco factores. Creemos firmemente que la colaboración entre centros educativos puede ser una respuesta eficaz y satisfactoria con la que conducirse en ese nuevo marco de situaciones y requerimientos.

Las soluciones, desde luego, también están en manos de los poderes públicos, de la Administración Educativa. Dejaremos, sin embargo, para otro momento cuál debería ser su papel, ya reiterado en múltiples ocasiones por nosotros mismos y por otros estudiosos, prácticos y por cualquier persona que tenga un afán por la mejora de la educación escolar pública.

Colaborar no es sino la acción de obrar conjuntamente con otro u otros con el propósito compartido de alcanzar un mismo fin. La acción de colaborar puede desarrollarse entre estamentos: profesorado, alumnado, padres y madres; entre servicios: 
Inspección, servicios psicopedagógicos de apoyo a los centros...; entre centros educativos, etc. En nuestro caso, la colaboración entre centros, objeto central de esta aportación, es un modo de trabajar de dos o más establecimentos escolares, compartiendo recursos, para alcanzar unos propósitos específicos durante un período de tiempo determinado, que tiene como características y requisitos principales los siguientes:

(i) es voluntario;

(ii) está establecido en términos de colegialidad, entre iguales, no existe predominio por parte de uno de los agentes;

(iii) está abierto a la participación de todos los estamentos de la comunidad educativa;

(iv) se basa en la lealtad y en la confianza recíprocas;

(v) implica, por tanto, un determinado planteamiento ideológico (ausencia de jerarquías, equidad, igualdad entre participantes, voluntad de transformación y mejora...) (Hall y Wallace, 1993);

(vi) supone, a diferencia de la simple cooperación, realizar en común, participativamente, el diseño de lo que se pretende alcanzar o desarrollar, acordar la metodología de trabajo y discutir y evaluar en común el proceso y los resultados.

Colaborar, desde el modelo que planteamos, no supone, por tanto, simplemente ayudar en una parte del proceso, mediante una participación episódica o a través de una relación de dependencia o subordinada.

Ahora bien ¿cuáles son las razones para colaborar?, ¿qué se pretende con ello?, ¿qué lo justifica?

No resulta nada nuevo constatar que la colaboración entre centros es uno de los factores clave relacionados con la mejora de los aprendizajes de sus alumnos. Así lo certifican múltiples estudios, como los ya clásicos de Purkey y Smith (1983); Stuart y Scott (1990), en los Estados Unidos; o los de Rutter y otros (1979) o Reynolds (1992), en el Reino Unido.

Por otra parte, la colaboración se justifica por una razón bien simple y de sentido común: la acción sinérgica suele ser más efectiva y eficaz que la acción individual o que la simple adición de acciones individuales. Mediante la colaboración parece más factible mejorar las ayudas pedagógicas que proporcionamos a nuestros estudiantes o, si se prefiere, para ofrecer una oferta educativa más completa y una educación más justa. Pero además, el hecho de colaborar ayudará, de manera más específica a:

- Analizar en común, entre centros, problemas que son comunes, con mayores y mejores criterios.

- Contribuir a la mejora de la imagen de la escuela pública, dignificándola, conseguir un reconocimiento y reforzar una identidad.

- Aumentar el autoconcepto y la autoestima entre el profesorado y el sentimiento de propiedad y de pertenencia respecto al centro escolar.

- Constituir agrupaciones, consorcios, colectivos, grupos con la clara finalidad de defender los valores que señalábamos en el primer párrafo. 
- Ejercer la autocrítica con un afán de mejora y, en su caso, proponer correcciones y soluciones a las situaciones extremas de miembros de nuestro propio colectivo: enseñantes negligentes, absentistas..)

- Sobrevivir mediante soluciones concurrentes atrayendo a más alumnos y a los mejores profesores. Propósito éste bien prosaico pero también justificado en épocas en que existe una natalidad baja, recursos financieros escasos o el derecho a elegir centro por parte de las familias. Sería, esta última, la razón más pragmática de todas las que estamos comentando y para nosotros, evidentemente, no la única ni la más importante aunque las políticas educativas que promueven algunas formas de gobierno podrían elevarlá a la categoría de justificación máxima.

- Responder, en suma, a la presión por la competitividad a con creatividad y con planteamientos solidarios.

A pesar de la importancia de estas razones, las prácticas de colaboración entre centros públicos en España son, por ahora, poco numerosas. No obstante sí que existen algunos antecedentes. En general, se trata de experiencias de trabajo en común que han surgido por el interés de obtener determinados resultados o beneficios en situaciones muy concretas. Casi siempre han estado relacionadas con contextos deficitarios. Uno de los ejemplos más claros es el de las escuelas unitarias e incompletas de ámbito rural. Como sabemos, los factores que caracterizan a estos centros (Antúnez y Gairín, 1996: 249-253) tienen que ver fundamentalmente con tres elementos: fuerte influencia del contexto físico y social sobre los enseñantes (ideología, valores y normas predominantes en el municipio: la cultura local, en suma); diversidad (de requerimientos, de expectativas, de prácticas educativas para atender la diversidad, etc.) y precariedad (no tanto de recursos materiales y personales, últimamente, pero sí, todavía, en la formación inicial de sus profesores y fuertes limitaciones al acceso a una formación permanente específica).

¿Qué han venido haciendo los profesores y profesoras que trabajan en esos centros para superar aquella problemática? En primer lugar, demostrar continuamente que son uno de los colectivos más activos y sensibilizados por los problemas de la educación que se desarrolla tanto dentro de la escuela como fuera de ella. $Y$, en segundo, aproximarse, constituir núcleos de trabajo común, intercambiar experiencias, constituir Claustros de Zona, Agrupaciones de Centros.

Otras experiencias de colaboración entre centros públicos fueron, en su momento, en los años setenta especialmente, las de los centros de personas adultas. Estaban también, en general, en contextos deficitarios: precariedad de recursos, ubicación errática o inestabilidad de sus enseñantes.

En la actualidad, la colaboración, más que un ejercicio recomendable deviene un requisito. Así se está concibiendo cada vez más entre colectivos que buscan respuestas nuevas y creativas ante requerimientos nuevos. Es el caso reciente, por ejemplo, de algunas propuestas, como las de la Federació de Moviments de Renovació Pedagògica de Catalunya (1996) que tratan de promover la colaboración entre centros públicos de primaria y de secundaria. Plantean la creación de zonas de enseñanza obligatoria (ZEO), constituidas por un centro de enseñanza secundaria y varios 
centros de primaria adscritos. El alumnado de estos centros pasaría directamente al centro de secundaria correspondiente.

Para ello, en estas ZEO se facilitarían y desarrollarían coordinadamente actuaciones como:

- traspaso de información de unos centros a otros: en relación a los alumnos, a las propuestas curriculares, a las modalidades de gestión...;

- actividades conjuntas entre el profesorado de los centros que forman la ZEO;

- elaboración de un Proyecto Educativo de Zona;

- creación de un organismo de coordinación para hacer posible el dispositivo.

\section{Un instrumento para analizar y promover la colaboración entre instituciones escolares.}

Iniciarse en el camino de la colaboración requiere de pautas para guiar las acciones. La herramienta que presentamos: Continuo para el análisis y la mejora de la colaboración entre centros escolares (cuadro núm. 1) pretende ser un instrumento de referencia, para que, a partir de él, los equipos de enseñantes o comunidades educativas que tengan interés puedan organizar y orientar sus acciones conducentes al desarrollo y la mejora de la colaboración.

Consta de cinco escalones o grados. Cada uno de ellos describe un propósito, desde el más simple y menos ambicioso: Posibilitar, fomentar y aumentar el conocimiento recíproco, al más complejo y deseable: Establecer redes de centros. En cada escalón se presentan algunos ejemplos de acciones concretas que pueden servir de pauta para orientar y guiar el paso de un grado al otro.

El instrumento quiere sugerir la idea, nada dogmática, que para alcanzar los grados superiores conviene que se ejerciten antes las acciones que comportan los escalones inferiores. $Y$ que, por otra parte, las actuaciones propias de los escalones superiores frecuentemente engloban a las de los precedentes. También puede ser útil para guiar ordenadamente un proceso de evaluación formativa interna y para diseñar acciones de mejora.

Como solemos hacer habitualmente, recomendamos que la herramienta sea adaptada, completada, modificada y, en suma, mejorada (no será muy difícil) por quienes la usen, con el fin de hacerla más útil en cada contexto escolar e institucional.

Veamos cuáles son esos escalones grados y algunos ejemplos de acciones propias de cada uno de ellos.

1‥ Posibilitar, fomentar y aumentar el conocimiento recíproco.

- intercambiar informaciones mediante la realización de visitas, aprovechando las coincidencias durante el desarrollo de actividades conjuntas de formación permanente del profesorado (FPP);

- desarrollar contactos formales e informales fuera del centro: aprovechando reuniones zonales entre directivos, entre profesores; aprovechando encuentros con motivo de acontecimientos, celebraciones...; 
- cursar invitaciones a otros centros para que sus enseñantes puedan estar presentes en el nuestro: observando, participando pasivamente en actividades del centro...;

- promover e incrementar los contactos motivados por el traspaso de informaciones, p. e.: noticias profesionales, novedades curriculares, legislativas, relativas al mapa escolar, a problemáticas escolares propias del municipio, relacionadas con un sector de profesionales (personal no numerario, profesorado especialista en un área concreta...), de la zona escolar; problemáticas laborales: luchas sindicales, huelgas...,

- esporádicamente y, más adelante, sistemáticamente se puede aumentar también el conocimiento recíproco intercambiando experiencias organizativas, didácticas o de orientación escolar, personal o profesional: a partir de un plan de trabajo establecido en común, mediante encuentros, jornadas, escuelas de verano o aprovechando los dispositivos de FPP disponibles en la zona (Centros de Profesores, de Recursos, Institutos de Ciencias de la Educación...).

Este primer paso supone también desarrollar procesos para darnos a conocer más y mejor a la comunidad escolar en la que nos encontramos (Antúnez, 1997). Hacer bien lo que hacemos y saberlo explicar constituyen, sin duda, las primeras acciones que hay que promover con decisión, y no para competir necesariamente ya que en muchos casos la competición no ha lugar (imaginemos, por ejemplo, un municipio pequeño en el que sólo existe un centro escolar).

\section{$2^{2}$. Denunciar situaciones escolares injustas y reivindicar mejoras $y$ el cumplimiento de las leyes.}

Un paso muy importante para aglutinar docentes y centros consiste en unirse para reivindicar el cumplimiento justo de las normativas que afectan a la educación escolar, especialmente las que regulan:

A la admisión y matriculación de alumnos en los centros que acogen las etapas educativas obligatorias, procurando que ambos procesos se desarrollen de manera que no se discrimine a algunos centros y se respeten los principios constitucionales de publicidad o de justicia;

A la contratación del profesorado en los centros privados concertados, dada la desidia de los sindicatos en relación a este aspecto;

Las acciones conducentes a reivindicar razonablemente mejoras y recursos, sobre todo en la FPP, en centros y en zonas escolares son también útiles para promover procesos de colaboración entre centros. Igualmente, la razonable pero decidida presión a la Administración Educativa para que desarrolle políticas que hagan posible:

- La puesta en marcha y aplicación de sistemas de evaluación adecuados internos y externos para que cada centro sea juzgado según sus circunstancias particulares: posibilidades, condiciones, restricciones, y recursos reales.

- retirar los velos, ayudar a esclarecer ¿qué se esconde realmente detrás de un centro "de prestigio", de renombre o que merece un buen crédito?; 
- intervenir decididamente en casos extremos (desidia profesional de los docentes, mal uso de los recursos...);

- evitar conductas de connivencia en sus servicios, lesivas para la escuela pública;

- proporcionar recursos que compensen las desigualdades en las zonas y a los centros que acogen poblaciones escolares en situaciones difíciles.

Otras acciones propias de este segundo nivel serían:

- Dar a conocer a la opinión pública, a la sociedad, cuál es la situación real de la escuela pública, desde un propósito nada corporativista y sí de mejora tratando de responder cada vez mejor a los derechos y necesidades de nuestros estudiantes.

- Evitar los efectos negativos de la especialización. Sería el caso de los centros que al especializarse en la atención al alumnado de clases sociales desfavorecidas o a los estudiantes con necesidades educativas especiales, proyectan una imagen poco atractiva aún siendo eficaces y laboriosos.

\section{Compartir recursos.}

Este tercer paso en el camino de la colaboración no sugiere ni plantea la idea desarrollar tareas de suplencia. Es decir, no se trata de cubrir, a base de esfuerzos personales, las tareas y prestaciones que son responsabilidad de los poderes públicos.

Nos referimos a que, sin dejar de reivindicar lo que creemos que es justo, se puede profundizar en la colaboración compartiendo, intercambiando o usando de manera rotativa recursos del tipo:

- profesores de apoyo, especialistas en áreas como Educación Física, Ed. Musical, Lengua Extranjera...;

- profesionales especialistas: pedagogo, psicólogo, administrador, asesores de FPP;

- material de uso didáctico (bibliográfico, audiovisual, de laboratorio...);

- espacios (gimnasio, sala de actos, áreas de usos diversos...);

- servicios internos (comedor, secretaría, transporte...);

- servicios externos de apoyo (Centros de Profesores, de Recursos, Equipos Multiprofesionales...);

\section{4 . Participar en proyectos comunes.}

En este nivel de colaboración encontraríamos acciones como las siguientes:

- desarrollo de tareas conjuntas de planificación, ejecución y evaluación del curriculum;

- constitución de Claustros de Zona;

- construcción y desarrollando proyectos y planes comunes no curriculares, p.e.: plan para la difusión de una mejor imagen de la escuela pública en el municipio; campañas para potenciar determinados valores (respeto y conser- 
vación del entorno: recogida selectiva de papel, de pilas...); representaciones artísticas; coordinación entre centros con el fin de favorecer el paso de alumnos de una etapa a otra, etc.

- desarrollo conjunto de acciones formativas propias de la FPP: seminarios, grupos de trabajo, proyectos de investigación en la acción...

- intercambios de estudiantes, viajes de estudios, etc.

\section{5‥ Establecer redes de centros.}

Constituir agrupaciones, consorcios y, en fin, redes de centros tiene como finalidad principal disponer de un dispositivo estructurado y estable que permita sistematizar, instaurar, formalizar, evaluar y mejorar las acciones señaladas en los escalones anteriores.

Los establecimientos escolares, constituidos y asociados en grupos de colaboración, podrán, además, conseguir el reconocimiento de los poderes públicos, ser protagonistas de la interlocución con estos poderes y también constituirse en grupos de presión.

Es necesario que las redes de centros dispongan de una instancia que desempeñe las tareas de coordinación.

Un primer paso para conocer los beneficios de las redes de colaboración entre centros que comparten la idea de la innovación es aprovechar mejor las que ya existen, como las que ofrecen los Movimientos de Renovación Pedagógica.

También puede ser motivador descubrir y conocer embriones de redes locales, si existen, o, más adelante, analizar cómo se organizan y qué desarrollan redes supranacionales del tipo Eurydice, ISIP, etc. Análogamente, convendrá analizar las experiencias y posibilidades de las redes de centros virtuales mediante apoyos de las nuevas tecnologías de la información.

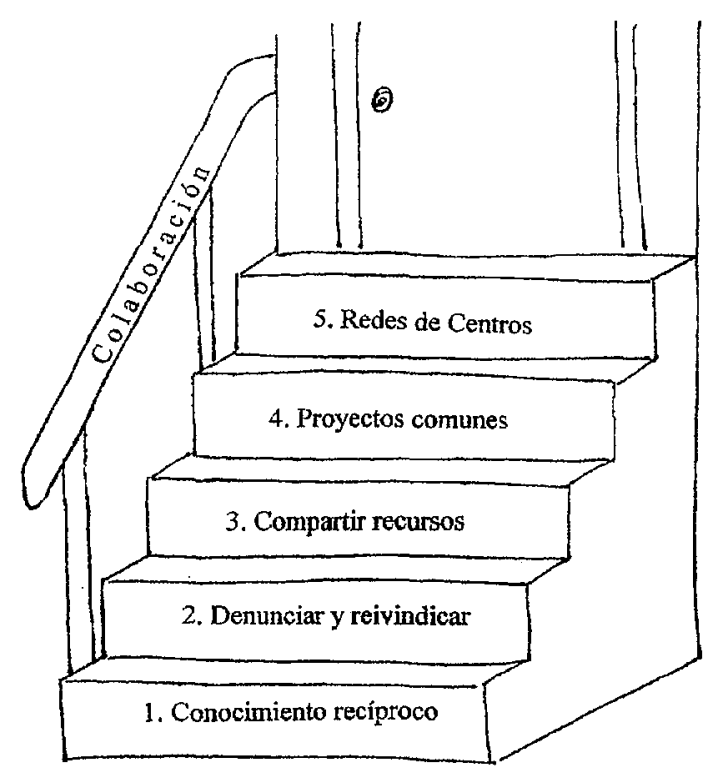

Cuadro $\mathrm{n}^{\mathrm{o}}$ 1. Continuo para el análisis y la mejora de la colaboración entre centros escolares 


\section{Algunas precauciones y requisitos}

Recorrer el continuo de la colaboración con la intención de responder, desde la escuela pública, a los nuevos retos y presiones requiere considerar algunas cautelas e implica, a la vez, cumplir con unas condiciones. Especialmente las siguientes.

\section{a) Reconsiderar, redefinir continuamente el concepto de calidad.}

Como decíamos al principio, todos parecemos abogar por que la educación sea de calidad. Ahora bien, ¿a qué nos estamos refiriendo cuando hablamos en esos términos? Ponerse de acuerdo sobre el concepto de calidad en educación es una tarea compleja. Tiene un significado polisémico. Depende de quién la defina, con qué intención, en relación a qué parámetros, respecto a qué objeto de estudio y, sobre todo, con qué intención se formula la noción y desde qué intereses.

En una cultura de colaboración y con el propósito de potenciar y dignificar la escuela pública, como ya hemos señalado en alguna otra ocasión, (Antúnez, 1994), la calidad, en tanto que propiedad o condición que otorgamos a la acción y al efecto de educar debería concebirse siempre en relación a unos determinados parámetros y no por efecto de la comparación o el contraste con otras acciones y efectos.

Calidad, pues, desde la colaboración que propugnamos, no sería, el término que designa situaciones o acciones que sobresalen o destacan en relación a otras de su mismo género o naturaleza (a la manera de la noción francesa de "exceller"). Sería, en cambio, la noción que designa la acción y el efecto que tienen la propiedad, condición o características que consideramos positivas en relación a unos determinados criterios o que cumplen unos determinados requisitos sobre los que existe un consenso y que son reconocidos como buenos respecto a unos determinados objetivos sobre los que también existe un consenso.

¿Cuáles podrían ser esos criterios o factores que identificasen una educación de calidad?. Creemos que, por una parte, existen unos criterios de carácter general y otros, de naturaleza más específica, sobre los que podríamos intentar llegar a acuerdos.

Los criterios o, si se prefiere, los requisitos generales de una educación de calidad deberíamos encontrarlos en los valores que defiende la Constitución. Así, podemos decir que existe calidad cuando la educación propugna y se desarrolla siguiendo como principios normativos a valores como: respeto, dignidad humana, justicia social, igualdad de derechos, libertad, participación, transparencia u otros principios democráticos.

Otros criterios más específicos de calidad podrían ser los que permiten la operativización de los criterios generales anteriores. Algunos ejemplos, entre otros, pueden ser:

- existencia de recursos suficientes (personales, materiales y funcionales) y capacidad para adecuar esos recursos a los fines que se pretenden,

- existencia de revisión y evaluación constantes (control interno y externo, control técnico y control social...), y capacidad para llevarlas a cabo con la intención de utilizar sus resultados para mejorar la teoría y la práctica, 
- existencia de cauces y órganos para posibilitar la participación en la gestión de los sistemas educativos y escolares,

- atención prioritaria hacia la formación inicial y permanente de los enseñantes en todas sus manifestaciones,

- existencia de actuaciones concretas en relación a la atención a la diversidad de alumnos, de profesores y de centros, etc.

- existencia de parcelas de autonomía suficientes (en los profesores, los centros y los sistemas educativos y escolares) y de capacidad para administrarlas,

b) Ejercitarse en la colaboración, comenzando el proceso mediante acciones en el propio centro.

Se trata de empezar por nuestra propia casa. Se pueden efectuar acciones coadyuvantes o propedéuticas a la colaboración entre centros:

- Desarrollando planes específicos de atención a las personas nuevas: enseñantes, padres y madres, estudiantes, que se incorporan cada año a la institución;

- Tratando de implicar a los padres y madres y a los estudiantes en la gestión del centro, definiendo en común el tipo de participación y de compromiso recíproco;

- Facilitando ta colaboración entre docentes. Muchos profesionales podemos buscar y encontrar la ocasión de observar el trabajo de nuestros colegas "in situ" y de participar en él. Observar y analizar en común el tipo de relaciones que establecemos con los alumnos y alumnas o la actividad en el aula; planificar sesiones de clase, elaborar y seleccionar materiales en común, evaluar en común..., son actuaciones factibles y casi siempre fructíferas y satisfactorias.

- Tratando de proyectar y de dar a conocer, como ya dijimos, una buena imagen institucional a la comunidad educativa.

\section{c) Poner énfasis en la planificación.}

Los acciones que comportan procesos de colaboración no deberían improvisarse. Es necesario, es un requisito, planificarlos en común. No es factible ningún proceso que implique trabajo colaborativo mínimamente coherente si no existe, por ejemplo: sincronización en cuestiones tan elementales como los horarios lectivos o de dedicación no docente entre los enseñantes de centros distintos que quieren trabajar juntos; una jornada similar (continuada o partida); o no se establece un día de la semana, para todos el mismo, dedicado a las actividades de formación permanente del profesorado, etc.

La implicación de las partes se conseguirá mejor si todas ellas tienen algún protagonismo en el momento de definir y diseñar qué es lo que se pretende hacer y si, además, pueden realizar sus aportaciones respecto a la metodología de trabajo que se seguirá para conseguirlo. 
d) Respetar la idiosincrasia y los contextos y culturas particulares.

Colaborar debe permitir, a la vez, mantener características, valores y rasgos propios. No tiene por qué implicar uniformización e incondicionalidad ciega. Debe permitir también la posibilidad de disponer y utilizar parcelas de libertad y grados de autonomía.

Por otra parte, el desarrollo de modelos y de experiencias no debería efectuarse por imitación o traslación directa de modelos o experiencias tomadas de otros contextos. Cada establecimiento escolar, cada zona educativa territorial tiene derecho a ser y a manifestarse diferente a los demás y conviene que fije sus propios caminos en función de las variables y los contextos particulares. No valen las mismas repuestas ante requerimientos y realidades diferentes.

\section{e) Desarrollar los procesos con progresión y parsimonia.}

La colaboración entre centros requiere de pequeños pasos, de ensayos y revisiones modestas. En muchas ocasiones supone cambios culturales profundos que difícilmente pueden llevarse a cabo en plazos breves. Aunque los procesos nunca son lineales ni irreversibles, conviene dotarse de marcos, de pautas y patrones de referencia, siempre necesarios. Nuestra propuesta de instrumento para quiar los procesos de colaboración entre centros tiene que ver con esa idea.

f) Manifestar una claro convencimiento e implicación por parte de los equipos directivos de los centros.

Todo proceso de innovación, además de condiciones favorables y un relevante apoyo externo, requiere de un motor interno que lo impulse. Las personas que desempeñan tareas directivas están especialmente llamadas para animarlos y facilitarlos. Esa asunción de cierto protagonismo no tiene por qué implicar estructuras jerárquicas que son, a menudo, la causa fundamental de las barreras a la colaboración.

\section{g) Empatía y honestidad.}

Un trabajo en colaboración que pretenda ser eficaz y satisfactorio implica la capacidad de comprender las diferencias, y también la capacidad de analizar los problemas y situaciones desde la perspectiva del otro. El diálogo continuo, abierto y sincero entre profesionales y entre instituciones deviene un requisito ineludible.

Por otra parte, la colaboración, requiere confianza, sinceridad y honestidad. El hecho de conocerse mejor debe servir para colaborar mejor no para advertir mejor los puntos fuertes y débiles de los demás y utilizar ese conocimiento con otras finalidades contrarias a aquel propósito. Traspasar alumnos problemáticos subrepticiamente a un centro vecino, también público, no es una práctica desconocida o inhabitual pero que no tendría cabida entre centros que colaboran entre sí. 


\section{h) Complementariedad y sinergía.}

La colaboración, tal como la concebimos, requiere de una complementariedad de roles y en las contribuciones de unos y otros. Cualquiera no debe hacer cualquier cosa, sino lo que es capaz de hacer mejor, desde un enfoque que trata de buscar en la concurrencia de actuaciones coordinadas el mayor efecto que la simple adición de acciones y efectos individuales.

Considerar estos dos factores supone la capacidad y la voluntad de reconceptualizar roles, metodología de trabajo, formas de operar y la actitud favorable hacia cambiarlos, si es preciso, en aras al beneficio colectivo.

\section{8. ¡Basta ya de pamplinas!}

Ahora bien, ¿qué podemos y deberíamos hacer desde los departamentos universitarios de Didáctica y Organización Educativa?. ¿Tenemos algún papel que desempeñar en estos procesos?

Como es bien notorio, a los pedagogos y pedagogas se nos achaca a menudo no aportar más que un aumento de voces, términos o nuevas expresiones para designar hechos fenómenos o artefactos de conocimiento común, y no tanto soluciones razonables y fundamentadas a los problemas de la educación.

Aumentar el caudal de voces y términos no responde siempre a la aparición o al aumento de novedades conceptuales o factuales o de producciones recientes o de inventos sino que bastante a menudo obedece a la más obstinada cultura de la "retórica pedagógica" pertinazmente instalada entre los que nos dedicamos al estudio de la educación.

Estas prácticas, a menudo estériles, están especialmente instaladas en muchos de nuestros departamentos universitarios. Nos dedicamos de describir los hechos (que, a menudo, sólo conocemos mediante fuentes indirectas); nos preocupa poner nombres a las cosas, etiquetar, clasificar, ubicar los acontecimientos en epígrafes, en modelos o en paradigmas. Nos ocupamos, sin embargo, algo menos de interpretar aquellos hechos, de conocer los porqués, sus causas o sus efectos. Finalmente, denotamos una despreocupación máxima cuando no tratamos de intervenir activamente por cambiar las cosas, con el fin de que mejoren, mediante prácticas educativas orientadas por unos determinados valores.

Tal vez ya vaya siendo hora de plantearnos algunas contribuciones algo más realistas y útiles. De lo contrario, estamos a punto de conseguir que la Organización Escolar se convierta en un terreno de estudio para diletantes, para deleite de ociosos, en un artificio poético, en un toreo de salón, en suma, meramente decorativo y sin riesgos. Considero, modestamente, y con todos los respetos, que no hacemos lo suficiente para promover ayudas a los compañeros y compañeras que están en la práctica diaria en los centros escolares no universitarios. Nosotros también estamos llamados a colaborar, a entrar en el juego, con ellos desempeñando nuestro papel (Nicholls, 1997).

Creo que, en relación a la colaboración, deberíamos asumir compromisos personales y departamentales conducentes a: 
a) Tratar de aumentar nuestra presencia o nuestra influencia en las instancias desde las que se puede incidir de manera más rápida y eficaz a la mejora de la educación escolar a través de la colaboración. Algunas de esas instancias están, desde luego, en el dispositivo, en el "aparato" de la Administración Educativa: sus subdirecciones, sus servicios (especialmente los de Inspección y de FPP) o sus secciones.

b) Orientar y abrir decididamente nuestro campo de acción hacia la FPP si, de verdad, queremos incidir directamente o a plazo corto en las prácticas reales de los centros escolares.

c) Conocer el medio. Para sugerir, para proponer, para ayudar a reflexionar hay que bajarse a la arena: a los centros, a los claustros, a las zonas escolares. No como "expertos infalibles", ¿es que alguien lo es?, sino como mediadores, como facilitadores. Salir de los despachos y de las sedes de los Departamentos y no pasarse el día especulando en ellos.

d) Promover y desarrollar programas de investigación en la acción donde tengan cabida y un tratamiento preferente los estudios vinculados a la práctica en las aulas y en los centros; codo con codo con las personas que están ellos y en los que éstas tengan un claro protagonismo y una oportunidad para su promoción y desarrollo personal y profesional.

e) Mejorar, en lo posible, los programas de prácticas de nuestros alumnos en establecimientos escolares para aumentar así el conocimiento recíproco y perfeccionar nuestras aportaciones.

f) Aplicar, en fin, nuestras propias prédicas a nuestra realidad institucional.

Tal vez merezca la pena hacer un esfuerzo entre todos e intentarlo.

\section{Referencias}

ÁNGULO, F. (1995) "El Neoliberalismo o el surgimiento del mercado educativo", en KIKIRIKÏ, núm. 35 (Dossier). Págs. 25-33

ANTÚNEZ, S. (1994): "La autonomía de los centros escolares, factor de calidad educativa y requisito para la innovación", en Revista de Educación, núm. 304. MayoAgosto. Págs 81-111.

ANTÚNEZ, S. (1997) "¿Márketing Escolar?, en AULA de Innovación Educativa, núm. 58, enero; págs. 57-59.

ANTÚNEZ, S. (1997): "Proyectar una buena imagen, ¿cómo nos damos a conocer?: un instrumento para la evaluación", en AULA de Innovación Educativa, núm. 58, enero; págs. 68-69.

CRUZ, J. E. (1996): "Educación pública: o competitiva o marginal (I)", en Escuela Española, núm. 3.279, 23 de mayo; pág. 2

CRUZ, J. E. (1996): "Educación pública: o competitiva o marginal (II)", en Escuela Española, núm. 3.280, 30 de mayo; pág. 2

HALL, V. y WALLACE, M. (1993): "Collaboration as a Subversive Activity: a professional response to externally imposed competition between schools?", School Organisation, Vol. 13, núm. 2 Págs. 101-117. 
FEDERACIÓ DE MOVIMENTS DE RENOVACIÓ PEDAGÓGICA DE CATALUNYA (1996): "Una proposta de coordinació entre els centres educatius públics de primària $\mathrm{i}$ secundària", en Temes de Renovació Pedagógica (separata), diciembre.

LE MONDE DE L'ÉDUCATION (1995): "BAC. Les résultats, lycée par lycée". Cuaderno suplementario del M 2172222 , enero.

LÓPEZ RUPÉREZ, F. (1997): "Una escuela pública de calidad", en Escuela Española, núm. 3.321 de 8 de mayo. Pág. 2

MACBETH, A., MC CREATH, D. y AITCHISON, J. (1995): Collaborate or Compete?. London. Falmer Press.

NICHOLLS, G. (1997): Collaborative Change in Education. London. Kogan Page.

PURKEY, S. y SMITH, M. (1983): "Effective Schools: a review", en The Elementary School Journal, núm. 85, págs. 353-390.

REYNOLDS, D. (Ed.) (1992): School Effectiveness: Research, Policy and Pratice. London. Ed. Cassell.

RUTTER, M., MAUGHAN, B., MORTIMORE, P., OUSTON, J. Y SMITH, A. (1979): Fifteen Thousand Hours: Secondary School and their Effects on Children. London. Ed. Open Books.

STUART, S. y SCOTT, J. (Eds.) (1990): The Collaborative School. Oregon. ERIC Clearinghouse on Educational Management. National Association of Secondary School Principals 
